Research Article

\title{
On Metrization of the Topologies Induced by Fuzzy Metrics
}

\author{
Jianrong $\mathrm{Wu} \mathbb{D}^{1}$ and Hao Yang ${ }^{1,2}$ \\ ${ }^{1}$ College of Mathematics and Physics, Suzhou University of Science and Technology, Suzhou, Jiangsu 215009, China \\ ${ }^{2}$ Department of Mathematics, Nantong Institute of Technology, Nantong, Jiangsu 226002, China
}

Correspondence should be addressed to Jianrong Wu; jrwu@mail.usts.edu.cn

Received 22 April 2020; Revised 21 June 2020; Accepted 26 June 2020; Published 21 July 2020

Academic Editor: Ljubisa Kocinac

Copyright ( 2020 Jianrong Wu and Hao Yang. This is an open access article distributed under the Creative Commons Attribution License, which permits unrestricted use, distribution, and reproduction in any medium, provided the original work is properly cited.

In this paper, we focus on finding the metric functions in a fuzzy metric space. After introducing the concept of a stratified function in a fuzzy metric space, we prove that the topology generated by the family of stratified functions coincides with the topology generated by the fuzzy metric. Moreover, we get the concrete form of metric function under some special conditions.

\section{Introduction}

Since Zadeh [1] first proposed fuzzy set theory in 1965, many researchers have defined concepts of fuzzy metric spaces and studied their properties in different ways [2-4]. Inspired by the notion of probabilistic metric spaces, Kramosil and Michalek [5] in 1975 introduced the notion of fuzzy metric, a fuzzy set in the Cartesian product $X \times X \times R$ satisfying certain conditions. Later, George and Veeramani [6] used the concept of continuous $t$-norms to modify this definition of fuzzy metric space and showed that every fuzzy metric space generates a Hausdorff first-countable topology. So far the GV-fuzzy metric theory has been developed by many researchers. Many important topics about the classical metric spaces were developed to fuzzy metric spaces. In this process, it was found that the theory of fuzzy metric was very different from the classical theory of metric. For example, Gregori and Romaguera [7] proved that there exists a GVfuzzy metric space which is not completable. Gregori and Romaguera [8] characterized the class of completable strong fuzzy metric spaces. Recently, some good related results about fixed point in fuzzy metric spaces were introduced. For example, one can see the works [9-11]. Meanwhile, the fuzzy metrics have been applied to domain theory, color image processing, and analysis of algorithms (see [12-17]).

In 2000, Gregori and Romaguera [18] obtained a somewhat surprising stronger result. They proved that every GV-fuzzy metric generates a metrizable topology. This important result connects the GV-fuzzy metric and the classical metric. However, the form of the metric function has not been explored in existing literature. It is just the main goal of the present paper.

In this paper, we first introduce the concept of a stratified function in a fuzzy metric space which is slightly different from the GV-fuzzy metric space and show that the topology induced by the family of stratified functions is compatible with the metrizable topology. Then, under some special conditions, we give the concrete metric function whose topology coincides with the metrizable topology.

The structure of the paper is as follows. In the next section, we give the preliminary notions on fuzzy metrics, with which we deal. In Section 3, we show our main results. Finally, we give our concluding remarks in Section 4.

\section{Preliminaries}

In this section, we first introduce some basic concepts and properties of fuzzy metric spaces.

Definition 1 (see [19]). A binary operation $*$ : $[0,1] \times$ $[0,1] \longrightarrow[0,1]$ is a continuous $t$-norm if it satisfies the following conditions:

(1) $*$ is associative and commutative

(2) $*$ is continuous

(3) $a * 1=a$ for each $a \in[0,1]$ 
(4) $a * b \leq c * d$ whenever $a \leq c$ and $b \leq d$ with $a, b, c, d \in[0,1]$

The following continuous $t$-norms are used in this paper:

$$
\begin{aligned}
& a \Delta_{1} b=\max \{a+b-1,0\}, \\
& a \Delta_{2} b=a b, \\
& a \Delta_{3} b=\min \{a, b\} .
\end{aligned}
$$

Also, we say the $t$-norm $\Delta t$ is stronger than the $t$-norm $\Delta^{\prime \prime}$ if $a \Delta^{\prime} b \geq a \Delta^{\prime \prime} b$ for all $a, b \in[0,1]$. In such case, we denote it as $\Delta^{\prime} \geq \Delta^{\prime \prime}$. It is easy to see that $\Delta_{3} \geq \Delta_{2} \geq \Delta_{1}$.

In the sense of Gregori and Veeramani [6], a GV-fuzzy metric is defined by the follows.

Definition 2. Let $X$ be a nonempty set and $*$ be a continuous $t$-norm. A fuzzy metric $M$ on the set $X$ is a mapping $M$ : $X^{2} \times(0, \infty) \longrightarrow(0,1]$ satisfying the following conditions: for all $x, y, z \in X, s, t>0$ :

$$
\begin{aligned}
& (\mathrm{GV} 1) M(x, y, t)>0, \\
& (\mathrm{GV} 2) M(x, y, t)=1 \text { if and only if } x=y, \\
& (\mathrm{GV} 3) M(x, y, t)=M(y, x, t), \\
& (\mathrm{GV} 4) M(x, y, t) * M(y, z, s) \leq M(x, z, t+s), \\
& (\mathrm{GV} 5) M(x, y, \cdot):(0, \infty) \longrightarrow(0,1] \text { is continuous. }
\end{aligned}
$$

If $M$ is a GV-fuzzy metric on $X$, then the 3-tuple $(X, M, *)$ is said to be a GV-fuzzy metric space. In that case, if confusion is not possible, we call $X$ a GV-fuzzy metric space for short. The following is a well-known result.

Lemma 1 (see [20]). $M(x, y, \cdot)$ is increasing for all $x, y \in X$. Gregori and Veeramani proved in [7] that every GV-fuzzy metric $M$ on $X$ generates a topology $\tau_{M}$ which has as a base

$$
\left\{B_{M}(x, r, t): x \in X, r \in(0,1), t>0\right\},
$$

where

$$
B_{M}(x, r, t)=\{y \in X: M(x, y, t)>1-r\},
$$

for all $x \in X, r \in(0,1)$, and $t>0$. They proved that for each $x \in X$, the family $\left\{B_{M}(x,(1 / n),(1 / n)): n \in \mathbf{N}\right\}$ is a local base at $x$. A sequence $\left\{x_{n}\right\}$ in $\left(X, \tau_{M}\right)$ converges to $x \in X$ if and only if $\lim _{n \rightarrow \infty} M\left(x_{n}, x, t\right)=1$ for all $t>0$. Also, by using Kelley metrization lemma [21], they also proved that $\tau_{M}$ is a metrizable topology.

\section{Main Results}

First, we introduce the concept of a stratified function in a GV-fuzzy metric space.

Definition 3. $(X, M, *)$ is a GV-fuzzy metric space. Let $r \in(0,1)$ and $x, y \in X$; set

$$
d_{r}(x, y)=\inf \{t>0: M(x, y, t)>1-r\} .
$$

Then, $d_{r}$ is called a $r$-stratified function with respect to $(X, M, *),\left\{d_{r}: 0<r<1\right\}$, the family of stratified functions.
To avoid the occurrence of the empty set, by a fuzzy metric in the rest of this paper, we mean a GV-fuzzy metric satisfying

$$
\text { (GV6) } \lim _{t \longrightarrow \infty} M(x, y, t)=1, \quad \forall x, y \in X .
$$

Lemma 2. Let $(X, M, *)$ be a fuzzy metric space, $r \in(0,1)$, $t>0, x, y \in X$. Then,

(1) For any $\lambda>d_{r}(x, y), M(x, y, \lambda)>1-r$

(2) The function $d_{r}$ is decreasing with respect to $r \in(0,1)$

(3) $B_{M}(x, r, t)=N_{r}(x, t)$, where

$$
N_{r}(x, t)=\left\{y \in X: d_{r}(x, y)<t\right\} .
$$

(4) The function $M(x, y, \cdot)$ is strictly increasing for the fixed points $x, y \in X$, if and only if for any $r \in(0,1)$,

$$
d_{r}(x, y)=\inf \{t>0: M(x, y, t) \geq 1-r\} .
$$

Proof

(1) Let $\lambda>d_{r}(x, y)$. From Definition 3, there exists $0<t<\lambda \quad$ such that $M(x, y, t)>1-r$. So, $M(x, y, \lambda)>1-r$.

(2) It follows from Lemma 1 directly.

(3) Let $y \in B_{M}(x, r, t)$, that is, $M(x, y, t)>1-r$. Since $M(x, y, \cdot)$ is continuous and increasing, there exists $0<t_{1}<t$ such that $M\left(x, y, t_{1}\right)>1-r$. From (5), we know $d_{r}(x, y) \leq t_{1}<t$. So, $y \in N_{r}(x, t)$. From the arbitrariness of $y$, we know that

$$
B_{M}(x, r, t) \subseteq N_{r}(x, t) .
$$

Let $z \in N_{r}(x, t)$; then, $d_{r}(x, z)<t$. From (5), there exists $0<t_{2}<t$ such that $M\left(x, z, t_{2}\right)>1-r$. Therefore, $\quad M(x, z, t) \geq M\left(x, z, t_{2}\right)>1-r$. So, $z \in B_{M}(x, r, t)$. From the arbitrariness of $z$, we know that $N_{r}(x, t) \subseteq B_{M}(x, r, t)$.

(4) Suppose that (8) holds; however, $M(x, y, \cdot)$ is not strictly increasing. Then, there exist $t_{1}, t_{2} \in\{t>0$ : $0<M(x, y, t)<1\}$ such that $t_{1}<t_{2}$ and $M(x, y, \cdot) \equiv 1-r_{0}$ on $\left[t_{1}, t_{2}\right]$. Thus,

$$
\begin{gathered}
\sup \left\{t>0: M(x, y, t) \leq 1-r_{0}\right\} \geq t_{2}>t_{1} \\
\quad \geq \inf \left\{t>0: M(x, y, t) \geq 1-r_{0}\right\} .
\end{gathered}
$$

It is easy to see that

$$
\begin{aligned}
& \sup \left\{t>0: M(x, y, t) \leq 1-r_{0}\right\} \\
& \quad=\inf \left\{t>0: M(x, y, t)>1-r_{0}\right\} .
\end{aligned}
$$

Therefore, $\quad d_{r_{0}}(x, y)>\inf \left\{t>0: M(x, y, t) \geq 1-r_{0}\right\}$, which conflicts with (8).

Conversely, suppose $M(x, y, \cdot)$ is strictly increasing. Let

$$
\begin{aligned}
t_{0} & =d_{r}(x, y) \\
& =\inf \{t>0: M(x, y, t)>1-r\} .
\end{aligned}
$$


Obviously, $\inf \{t>0: M(x, y, t) \geq 1-r\} \leq t_{0}$. If $\inf \{t>0:$ $M(x, y, t) \geq 1-r\}<t_{0}$, then there is $0<t_{2}<t_{0}$ such that $M\left(x, y, t_{2}\right) \geq 1-r$, so $M\left(x, y, t_{0}\right)>1-r$. Since $M(x, y, \cdot)$ is left continuous at $t_{0}$, there is $\delta>0$ such that $M\left(x, y, t_{0}-\delta\right)>1-r$, which conflicts with the definition of $t_{0}$. Thus, $\inf \{t>0: M(x, y, t) \geq 1-r\} \geq t_{0}$. So,

$$
\begin{aligned}
d_{r}(x, y) & =t_{0} \\
& =\inf \{t>0: M(x, y, t)>1-r\} .
\end{aligned}
$$

Now, from Lemma 2 (3), it is easy to see that the topology $\tau_{M}$ can be induced by the family of stratified functions. That is, we obtain the following theorem.

Theorem 1. Let $D=\left\{d_{r}: 0<r<1\right\}$ be the family of stratified functions with respect to a fuzzy metric space $(X, M, *)$, $N_{r}(x, t)$ be defined by (8), and

$$
B_{x}=\left\{N_{r}(x, t): r \in(0,1), t>0\right\} .
$$

Then,

(1) $B_{x}$ is a base of neighborhoods at $x \in X$.

(2) The topology $\tau_{D}$ generated by $\left\{B_{x}: x \in X\right\}$ coincides with the topology $\tau_{\mathrm{M}}$.

Generally, a stratified function is not a pseudometric. In fact, we have the following result.

Theorem 2. Let $(X, M, *)$ be a fuzzy metric space. A stratified function $d_{r}(r \in(0,1))$ is a pseudometric on $X$ if and only if $M$ satisfies the following condition: for any $x, y, z \in X, t_{1}, t_{2}>0$, if $M\left(x, z, t_{1}\right)>1-r, M\left(z, y, t_{2}\right)>$ $1-r$, then

$$
M\left(x, y, t_{1}+t_{2}\right)>1-r .
$$

Proof. For any $r \in(0,1)$, it is obvious that $d_{r}(x, y) \geq 0$, $d_{r}(x, y)=d_{r}(y, x)$, and $d_{r}(x, y)=0$ when $x=y$. Thus, to complete the proof, we only have to prove that $d_{r}(x, y) \leq d_{r}(x, z)+d_{r}(z, y)$ if and only if $M$ satisfies condition (15).

Sufficiency. For any $\varepsilon>0$, from Lemma 2 (1), we obtain

$$
\begin{aligned}
& M\left(x, z, d_{r}(x, z)+\frac{\varepsilon}{2}\right)>1-r, \\
& M\left(z, y, d_{r}(z, y)+\frac{\varepsilon}{2}\right)>1-r .
\end{aligned}
$$

From (15), we have

$$
M\left(x, y, d_{r}(x, z)+d_{r}(z, y)+\varepsilon\right)>1-r .
$$

Therefore,

$$
d_{r}(x, y) \leq d_{r}(x, z)+d_{r}(z, y)+\varepsilon .
$$

From the arbitrariness of $\varepsilon>0$, we know $d_{r}(x, y) \leq$ $d_{r}(x, z)+d_{r}(z, y)$.
Necessity. Suppose that $M\left(x, z, t_{1}\right)>1-r, M\left(z, y, t_{2}\right)>$ $1-r$. By (GV5), there exists $\delta>0$ such that

$$
\begin{aligned}
& M\left(x, z, t_{1}-\delta\right)>1-r, \\
& M\left(z, y, t_{2}-\delta\right)>1-r .
\end{aligned}
$$

So, $d_{r}(x, z) \leq t_{1}-\delta$ and $d_{r}(z, y) \leq t_{2}-\delta$. Since $d_{r}(x, y) \leq$ $d_{r}(x, z)+d_{r}(z, y)$, we have $d_{r}(x, y) \leq t_{1}+t_{2}-2 \delta<t_{1}+t_{2}$. By the definition of $d_{r}(x, y)$, there exists $t_{0}<t_{1}+t_{2}$ such that $M\left(x, y, t_{0}\right)>1-r$. Thus, $M\left(x, y, t_{1}+t_{2}\right)>1-r$.

Remark 1. $M$ satisfies (15) if $*=\Delta_{3}$.

Now, we explore the metric which induces the topology $\tau_{M}$.

Definition 4. Let $R^{+}=[0, \infty)$. We call function $f: R^{+} \longrightarrow R^{+}$ satisfies

(i) The condition $C_{1}$, if $f(0)=0, f(t) \equiv 0$, and $f$ is increasing and continuous at 0 .

(ii) The condition $C_{2}$, if $f(0)=0, f(t)>0$ as $t>0$, $\lim _{t \rightarrow+\infty} f(t)=+\infty, f\left(t_{1}+t_{2}\right) \leq f\left(t_{1}\right)+f\left(t_{2}\right)$ for any $t_{1}, t_{2} \in R^{+}$, and $f$ is left continuous and increasing.

Theorem 3. $(X, M, *)$ is fuzzy metric space; the functions $K$ and $G$ satisfy the conditions $C_{1}$ and $C_{2}$, respectively. Define a function $d$ on $X \times X$ as

$$
\begin{array}{r}
d(x, y)=\inf \{s>0: \text { if } G(t)>s, \text { then } M(x, y, t)+K(s) \geq 1\}, \\
\forall x, y \in X .
\end{array}
$$

If one of the following conditions is satisfied:

(I) $M$ satisfies condition (15)

(II) $* \geq \Delta_{1}, \forall \mathrm{r}_{1}, \mathrm{r}_{2} \in[0, \infty)$

$$
\mathrm{K}\left(\mathrm{r}_{1}\right)+\mathrm{K}\left(\mathrm{r}_{2}\right) \leq \mathrm{K}\left(\mathrm{r}_{1}+\mathrm{r}_{2}\right) \text {, }
$$

then $\mathrm{d}$ is a metric on $\mathrm{X}$.

Proof. First, we prove the following fact: if

$$
G(t)>r>d(x, y)
$$

then

$$
M(x, y, t) \geq 1-K(r) .
$$

In fact, if $G(t)>r>d(x, y)$, from the definition of $d$, we obtain there exists $0<s<r$ such that if $G(t)>s$, then $M(x, y, t)+K(s) \geq 1$. Therefore, if $G(t)>r$, then $G(t)>s$, and hence, $M(x, y, t)+K(r) \geq M(x, y, t)+K(s) \geq 1$. That is, $M(x, y, t) \geq 1-K(r)$.

Next, we prove $d$ is a metric on $X$, that is, $d$ satisfies the following properties: for any $x, y, z \in X$,

$$
\begin{aligned}
& \left(M_{1}\right) d(x, y) \geq 0, d(x, y)=d(y, x) \\
& \left(M_{2}\right) d(x, y)=0 \text { if and only if } x=y \\
& \left(M_{3}\right) d(x, y) \leq d(x, z)+d(z, y)
\end{aligned}
$$


The conclusion $\left(M_{1}\right)$ is obvious.

For the conclusion $\left(M_{2}\right)$, it is easy to see that $d(x, y)=0$ if $x=y$. Now, we suppose $d(x, y)=0$; however, $x \neq y$. Then, there exists $t_{0}>0$ such that $M\left(x, y, t_{0}\right) \neq 1$, that is, $M\left(x, y, t_{0}\right)<1$. Since $K$ is continuous at 0 , there exists $0<r_{0}<G\left(t_{0}\right)$ such that $K\left(r_{0}\right)<1-M\left(x, y, t_{0}\right)$. This is in direct contradiction to (23). Thus, $d(x, y)=0$ implies that $x=y$.

To prove $\left(M_{3}\right)$, we take $r_{1}>d(x, z)$ and $r_{2}>d(z, y)$ arbitrarily. From (23), we know

(i) If $G(t)>r_{1}$, then

$$
M(x, z, t) \geq 1-K\left(r_{1}\right) \geq 1-K\left(r_{1}+r_{2}\right) .
$$

(ii) If $G(t)>r_{2}$, then

$$
M(z, y, t) \geq 1-K\left(r_{2}\right) \geq 1-K\left(r_{1}+r_{2}\right) .
$$

Now, suppose that $G(t)>r_{1}+r_{2}$. Let

$$
t_{0}=\inf \left\{s: 0<s \leq t, G(s)>r_{1}\right\} .
$$

Obviously, $t_{0} \leq t$. It is easy to prove that $G\left(t_{0}\right) \leq r_{1}$. In fact, if $G\left(t_{0}\right)>r_{1}$, from the left continuity of $G$, there exists $\eta>0$ such that $G\left(t_{0}-\eta\right)>r_{1}$. By the definition of $t_{0}$, we conclude that $t_{0} \leq t_{0}-\eta$, a contradiction. The fact $G\left(t_{0}\right) \leq r_{1}$ implies that $G\left(t-t_{0}\right) \geq G(t)-G\left(t_{0}\right)>r_{2}$. By the left continuity of $G$ again, we know there exists $\varepsilon>0$ such that $G\left(t-\left(t_{0}+\varepsilon\right)\right)=G\left(t-t_{0}-\varepsilon\right)>r_{2}$. By the definition of $t_{0}$, there exists $0<s_{1} \leq t$ such that $G\left(s_{1}\right)>r_{1}$ and $s_{1} \leq t_{0}+\varepsilon$. Noting that $G$ is increasing, we obtain that $G\left(t_{0}+\varepsilon\right) \geq G\left(s_{1}\right)>r_{1}$. From (i) and (ii), we have

$$
\begin{aligned}
M\left(x, z, t_{0}+\varepsilon\right) & \geq 1-K\left(r_{1}\right) \geq 1-K\left(r_{1}+r_{2}\right) \\
M\left(z, y, t-\left(t_{0}+\varepsilon\right)\right) & \geq 1-K\left(r_{2}\right) \\
& \geq 1-K\left(r_{1}+r_{2}\right) .
\end{aligned}
$$

Combining conditions (I) and (II), we get $M(x, y, t) \geq$ $1-K\left(r_{1}+r_{2}\right)$.

By the definition of $d$, we know $d(x, y) \leq r_{1}+r_{2}$. Since $r_{1}>d(x, z)$ and $r_{2}>d(z, y)$, we obtain

$$
d(x, y) \leq d(x, z)+d(z, y)
$$

directly.

Theorem 4. $(X, M, *)$ is a fuzzy metric space. $M$ satisfies condition (15) or $* \geq \Delta_{1}$. Let

$$
\rho(x, y)=\inf \{a>0: M(x, y, a)+a \geq 1\}, \quad \forall x, y \in X .
$$

Then, $\rho$ is a metric on $\mathrm{X}$ and the topology $\tau_{\rho}$ induced by $\rho$ coincides with the topology $\tau_{\mathrm{M}}$.

Proof. Let $G(t)=t, K(t)=t, \forall t \geq 0$. Then, $K$ and $G$ satisfy the conditions $C_{1}$ and $C_{2}$, respectively. Besides, $K$ satisfies condition (21). From Theorem 3, we know that

$$
\begin{array}{r}
d(x, y)=\inf \{s>0: \text { if } t>s \text {, then } M(x, y, t)+s \geq 1\}, \\
\forall x, y \in X,
\end{array}
$$

is a metric on $X$. Thus, to show that $\rho$ is a metric, we only need to show $d(x, y)=\rho(x, y), \forall x, y \in X$. In fact, if $a>0$, $M(x, y, a)+a \geq 1$ and $t>a$, then $M(x, y, t)+a \geq$ $M(x, y, a)+a \geq 1$. Therefore,

$$
\begin{aligned}
\{a & >0: M(x, y, a)+a \geq 1\} \\
& \subseteq\{s>0: \text { if } t>s, \text { then } M(x, y, t)+s \geq 1\} .
\end{aligned}
$$

From (29) and (30), we get $\rho(x, y) \geq d(x, y)$. On the other hand, from (30), for any $\varepsilon>0$, there exists $s>0$ such that $s<d(x, y)+\varepsilon$ and $M(x, y, t)+s \geq 1$ when $t>s$. Thus,

$$
M(x, y, d(x, y)+\varepsilon)+s \geq 1
$$

and hence

$$
M(x, y, d(x, y)+\varepsilon)+d(x, y)+\varepsilon>1 .
$$

From (29), we get $\rho(x, y) \leq d(x, y)+\varepsilon$. From the arbitrariness of $\varepsilon>0$, we have $\rho(x, y) \leq d(x, y)$. Thus, $d(x, y)=\rho(x, y)$.

Let $\quad r \in(0,1), \quad x \in X$. We put $U(x, r)=$ $\{y \in X: \rho(x, y)<r\}$. It is easy to show that

$$
U(x, r) \subseteq B_{M}(x, r, r) \subseteq U(x, 2 r) .
$$

In fact, for any $y \in U(x, r)$, inf $\{a>0: M(x, y, a)+$ $a \geq 1\}<r$. Thus, there is $a>0$ such that $a<r$ and $M(x, y, a)+a \geq 1$. So, $\quad M(x, y, r)+r>1$, that is, $y \in B_{M}(x, r, r)$. Hence,

$$
U(x, r) \subseteq B_{M}(x, r, r) .
$$

On the other hand, for any $z \in B_{M}(x, r, r), M(x, z, r)+$ $r>1$. From (29), we get $\rho(x, z) \leq r<2 r$. So, $z \in U(x, 2 r)$. Hence,

$$
B_{M}(x, r, r) \subseteq U(x, 2 r) .
$$

And (34) holds, which implies that $\tau_{\rho}=\tau_{M}$ directly.

Lemma 3. $(X, M, *)$ is fuzzy metric space; the functions $K$ and $G$ satisfy the conditions $C_{1}$ and $C_{2}$, respectively. Then, the function $d$ defined by (20) can be represented as follows, $\forall x, y \in X$ :

$$
d(x, y)=\sup \{s>0 \text { : if } G(t)>s \text {, then } M(x, y, t)+K(s)<1\} \text {. }
$$

Proof. In fact, we only need to show that $\inf A=\sup B$, where

$$
\begin{gathered}
A=\{a>0: \text { if } G(t)>a, \text { then } M(x, y, t)+K(a) \geq 1\}, \\
B=\{b>0: \text { if } G(t)>b, \text { then } M(x, y, t)+K(b)<1\} .
\end{gathered}
$$

Take $a \in A, b \in B$ arbitrarily. Suppose $b>a$. If $G(t)>b$, then $G(t)>a$. From the definitions of $A$ and $B$, we obtain

$$
M(x, y, t)+K(a) \geq 1>M(x, y, t)+K(b) .
$$

This is in direct contradiction to the condition that $K$ is increasing. So, $a \geq b$, and hence, $\inf A \geq \sup B$.

Now, let $\inf A=\alpha, \sup B=\beta$. For any $\delta>0$, we know $\beta+\delta \notin B$, that is, $\beta+\delta \in A$, which implies that inf $A \leq \beta+\delta$. 
By the arbitrariness of $\delta$, we know that $\inf A \leq \beta=\sup B$. This completes the proof.

Theorem 5. ( $X, M, *)$ is a fuzzy metric space. If $M$ satisfies condition (15) or $* \geq \Delta_{1}$, then $\forall x, y \in X$ :

$$
\rho(x, y)=\sup \{t>0: M(x, y, t)+t<1\},
$$

where $\rho$ is defined by (29).

Proof. Let $\mathrm{G}(\mathrm{t})=\mathrm{t}, \mathrm{K}(\mathrm{t})=\mathrm{t}, \forall \mathrm{t} \geq 0$. Then, (40) follows from Lemma 3 directly.

\section{Conclusions}

In the present paper, we investigate some metric structures in a fuzzy metric space. Especially, we give the concrete form of metric function with respect to the metrizable topology for a fuzzy metric under two special cases. Based on the results in the paper, interesting future research studies about the related topics may be prospective. Moreover, the technique used in this paper is suggestive to discuss the related problem in the general case.

\section{Data Availability}

No data were used to support this study.

\section{Conflicts of Interest}

The authors declare that they have no conflicts of interest.

\section{Authors' Contributions}

Jianrong $\mathrm{Wu}$ was responsible for conceptualization, methodology, funding acquisition, and review and editing. Hao Yang contributed to formal analysis, investigation, and original draft preparation. All authors have read and agreed to the published version of the manuscript.

\section{Acknowledgments}

This study was supported by the National Natural Science Foundation of China under grant no. 11971343.

\section{References}

[1] L. A. Zadeh, "Fuzzy sets," Information and Control, vol. 8, no. 3, pp. 338-353, 1965.

[2] Z. Deng, "Fuzzy pseudo-metric spaces," Journal of Mathematical Analysis and Applications, vol. 86, no. 1, pp. 74-95, 1982.

[3] M. A. Erceg, "Metric spaces in fuzzy set theory," Journal of Mathematical Analysis and Applications, vol. 69, no. 1, pp. 205-230, 1979.

[4] O. Kaleva and S. Seikkala, "On fuzzy metric spaces," Fuzzy Sets and Systems, vol. 12, no. 3, pp. 215-229, 1984.

[5] I. Kramosil and J. Michalek, "Fuzzy metrics and statistical metric spaces," Kybernetika, vol. 11, pp. 336-344, 1975.

[6] A. George and P. Veeramani, "On some results in fuzzy metric spaces," Fuzzy Sets and Systems, vol. 64, no. 3, pp. 395-399, 1994.
[7] V. Gregori and S. Romaguera, "On completion of fuzzy metric spaces," Fuzzy Sets and Systems, vol. 130, no. 3, pp. 399-404, 2002.

[8] V. Gregori and S. Romaguera, "Characterizing completable fuzzy metric spaces," Fuzzy Sets and Systems, vol. 144, no. 3, pp. 411-420, 2004.

[9] V. Gupta, A. Kaushik, and M. Verma, "Some new fixed point results on V- $\psi$-fuzzy contraction endowed with graph," Journal of Intelligent \& Fuzzy Systems, vol. 36, no. 6, pp. 6549-6554, 2019.

[10] V. Gupta, M. Verma, and J. Kaur, "A new contraction and existence theorems on fuzzy metric space with a graph," Italian Journal of Pure and Applied Mathematics, vol. 43, pp. 717-729, 2020.

[11] M. Verma, R. K. Saini, and V. Gupta, "Fixed point theorem by altering distance technique in complete fuzzy metric spaces," International Journal of Computer Aided Engineering and Technology, vol. 13, no. 4, pp. 437-447, 2020.

[12] V. Gregori, S. Morillas, and A. Sapena, "Examples of fuzzy metrics and applications," Fuzzy Sets and Systems, vol. 170, no. 1, pp. 95-111, 2011.

[13] S. Morillas, V. Gregori, G. Peris-Fajarnés, and P. Latorre, "A new vector median filter based on fuzzy metrics," in Lecture Notes in Computer Science, vol. 3656, pp. 81-90, Springer, Berlin, Germany, 2005.

[14] S. Morillas, V. Gregori, G. Peris-Fajarnés, and P. Latorre, "A fast impulsive noise color image filter using fuzzy metrics," Real-Time Imaging, vol. 11, no. 5-6, pp. 417-428, 2005.

[15] S. Romaguera, A. Sapena, and P. Tirado, "The Banach fixed point theorem in fuzzy quasi-metric spaces with application to the domain of words," Topology and Its Applications, vol. 154, no. 10, pp. 2196-2203, 2007.

[16] J.-R. Wu and Z.-Y. Jin, "A note on ulam stability of some fuzzy number-valued functional equations," Fuzzy Sets and Systems, vol. 375, pp. 191-195, 2019.

[17] J.-R. Wu, X.-W. Kai, and J.-J. Li, "Atoms of monotone setvalued measures and integrals," Fuzzy Sets and Systems, vol. 304, no. 1, pp. 131-139, 2016.

[18] V. Gregori and S. Romaguera, "Some properties of fuzzy metric spaces," Fuzzy Sets and Systems, vol. 115, no. 3, pp. 485-489, 2000.

[19] B. Schweizer and A. Sklar, "Statistical metric spaces," Pacific Journal of Mathematics, vol. 10, no. 1, pp. 314-334, 1960.

[20] M. Grabiec, "Fixed points in fuzzy metric spaces," Fuzzy Sets and Systems, vol. 27, no. 3, pp. 385-389, 1988.

[21] J. L. Kelley, General Topology, Van Nostrand, New York, NY, USA, 1955. 